\title{
Intramuscular Sinusoidal Hemangioma with Masson's Lesion, Masquerading as Parotid Tumor
}

\section{Tanmoy Deb ${ }^{1}$}

\section{Introduction:}

\section{ABSTRACT}

Sinusoidal hemangioma is a rare variant of cavernous hemangioma, described first by Calonje and Fletcher, more frequently subcutaneous and commonly found on the extremities. Fine needle aspiration cytology report was indeterminate. In such cases preoperative diagnosis is difficult. Slowly growing mass is the usual presenting complaint.

\section{Case Report:}

A rare case of intramuscular sinusoidal hemangioma, with typical Masson's lesion masquerading as a parotid tumor, in middle aged woman.

\section{Discussion:}

Hemangiomas comprise 7\% of all benign tumors and they are benign proliferative vascular tumors characterized by increased endothelial cell turn over. They are usually superficial and easy to diagnose whereas intramuscular hemangiomas (IMH) are uncommon and deep seated and difficult to diagnose. Fifteen percent of the IMHs are found in the head and neck area and the most common muscle affected is the masseter.

Keywords:

Hemangioma; Masseter Muscle; Intravascular Papillary Endothelial Hyperplasia

$\mathrm{P}$ athologists Calonje and Fletcher, were the first to describe sinusoidal hemangioma in 1991. It was considered to be a subset of cavernous hemangioma. It shows a lobular architecture consisting of dilated interconnecting (sinusoidal), thin walled vascular channels lined by a single layer of endothelium. ${ }^{1}$ Intramuscular sinusoidal hemangioma (IMH), with typical Masson's lesion is a very rare benign tumour, constituting less than $1.0 \%$ of all haemangiomas. ${ }^{2} \mathrm{We}$ believe it is all the more rare on the face and parotid region.

\section{Case Report}

A 40 year old female patient presented to the ear nose

1 - Agartala Government Medical College, Agartala

\section{Corresponding author:}

Dr Tanmoy Deb

email: drtanmoy.deb@gmail.com and throat (ENT) outpatient department (OPD) of our hospital with complaint of a slowly growing swelling on the left side of her face, which she noticed for the first time about a year back. On palpation the tumor was $3 \mathrm{~cm} \times 2.5 \mathrm{~cm}$, ovoid, with smooth surface and firm in consistency, mobile located in the parotid region and very close the angle of the mandible. (Fig.1) The swelling was mildly tender and there was no local rise of temperature, pulsation or bruit. The swelling was not compressible. There was no other palpable swelling in the neck. The facial nerve, along with all other cranial nerves, was normal. The overlying skin was not fixed to the tumour. Her general examination was normal. Her preoperative routine tests like complete blood count, blood sugar, liver function and kidney function test etc were within normal limits. FNAC was done but was inconclusive as it drew a few adipocytes and blood cells. NCCT neck was inconclusive too said it did not look like lipoma. So it was decided that excision biopsy under general anesthesia would be the best way out.

Accordingly she was taken up for surgery under general 


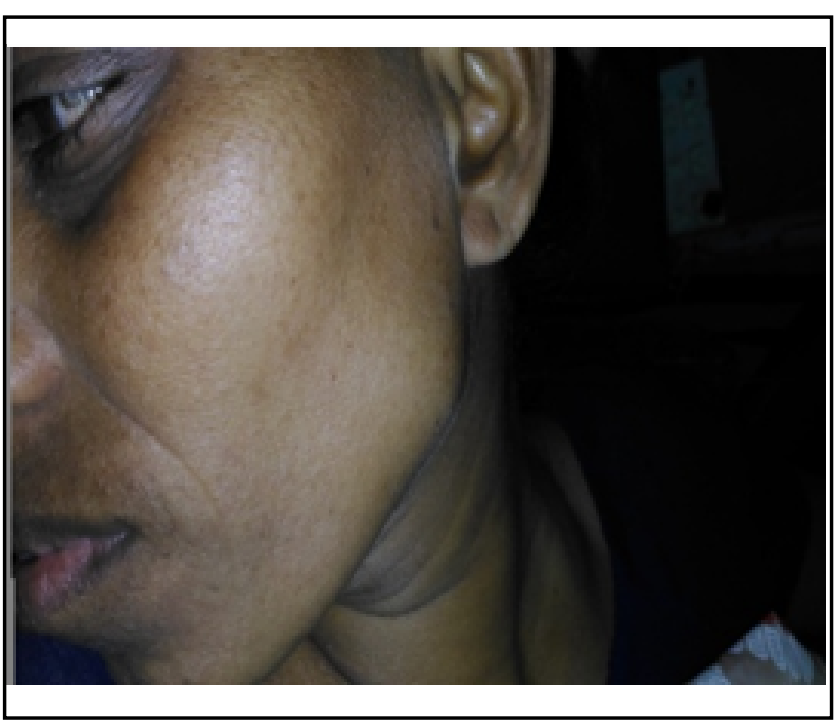

Fig. 1. Clinical picture of the tumor in the parotid region.

anesthesia with the superficial parotidectomy approach through a modified Blair incision. The skin flap was raised but the parotid tissue was found to be normal. The tumor, arising from the masseter muscle, was excised, taking adequate care not to damage the facial nerve, and was sent for histopathological examination (HPE). She was discharged from the hospital on the second post

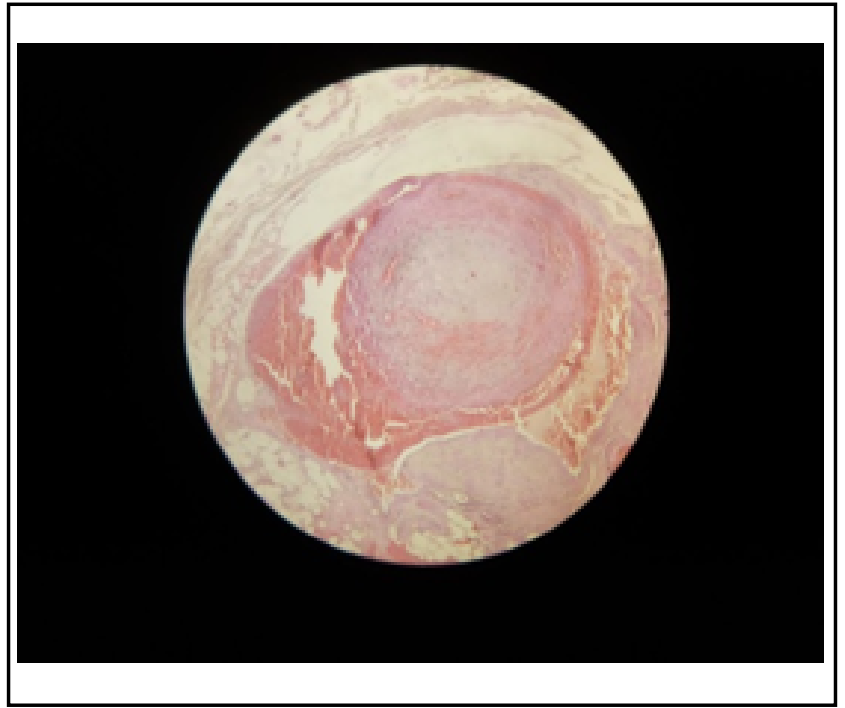

Fig. 3. Photomicrograph showing organizing thrombus within a blood vessel. (H\&E x 100)

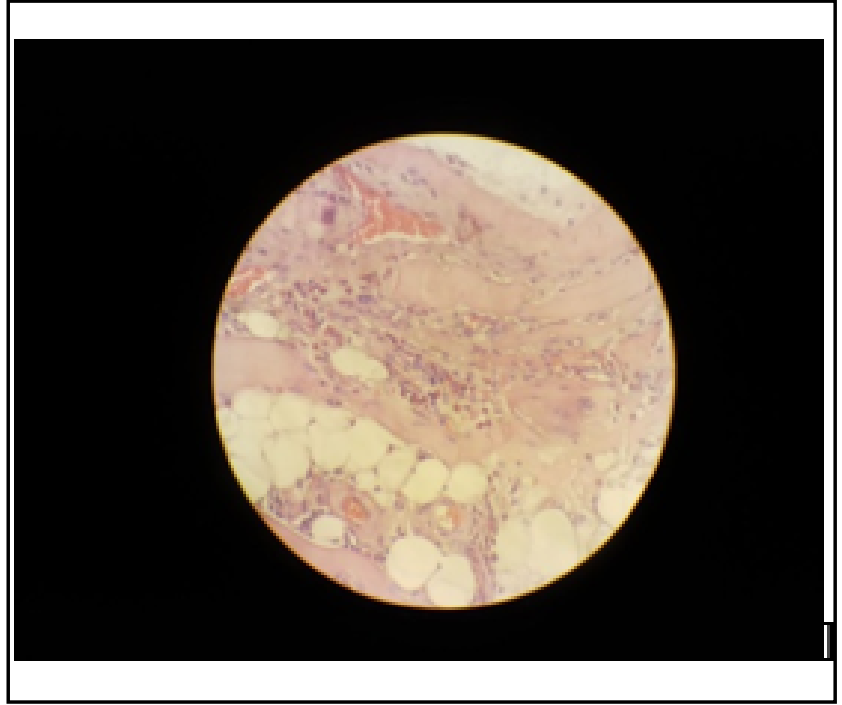

Fig. 2. Photomicrograph showing proliferating endothelial cells and adipocytes (H\&E $x$ 100)

operative day. The sutures were removed on the 7 th post operative day. The patient recovered uneventfully and the wound healed. On follow up visit up to post operative 4 months there was no recurrence. The HPE revealed the true nature of the tumor. As is evident in figures 2, 3 and 4 the tumor is a typical intramuscular sinusoidal hemangioma with typical Masson's lesion.

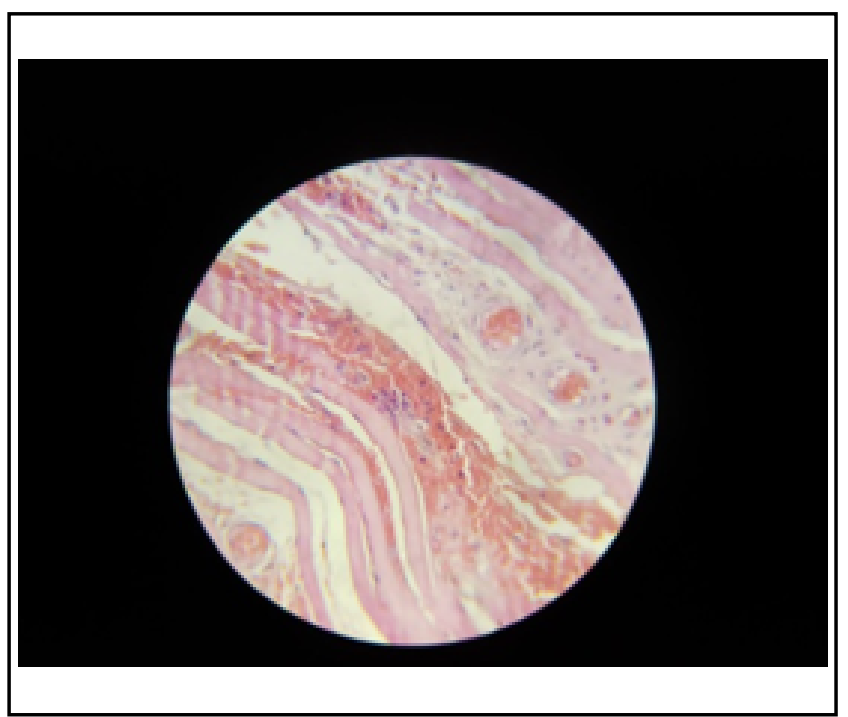

Fig. 4. Photomicrograph showing vascular spaces in between muscle bundles lined by single layer of endothelium. (H\&E x100) 


\section{Discussion}

Differential diagnosis: Intramuscular lipoma has a more indolent course and shows more of fat cells and very less vascular component. ${ }^{3}$ IMH can be differentiated from other vascular malformations of skeletal muscles by presence of complete or incomplete lining around the endothelial cells by smooth muscle cells and fibres forming a complete or partially matured wall, which is lacking in other hemangiomas. Angiosarcomas can be identified by presence nuclear and cellularpleomorphism, necrosis and mitotic figures. Intramuscular myxomas and granular cell tumors are characterized by presence of myxomatous and granular cell elements in the tissue. Angiomatosis involving skeletal muscle is another entity which can be very difficult to distinguish, histologically, from intramuscular haemangiomas except from some clinical parameters. Angiomatosis is usually congenital present from birth and involves extensive body areas involving skin muscle and even bone. Intramuscular hemangiomas are benign tumors with a small but definite risk of local recurrence. ${ }^{4}$

Hemangiomas comprise $7 \%$ of all benign tumors and they are benign proliferative vascular tumors characterized by increased endothelial cell turnover. They are predominantly superficial and easy to diagnose where as IMH are deep seated and are uncommon and difficult to diagnose. ${ }^{5} \mathrm{IMH}$ was first reported by Liston in $1843 .{ }^{6}$ Intramuscular sinusoidal hemangioma is a distinct type of cavernous hemangioma occurring within the skeletal muscles, constituting less than $1 \%$ of all haemangiomas. ${ }^{7}$

Fifteen percent of all IMHs occur in the head and neck area and the most common muscle affected, is the masseter muscle of the face followed by the trapezius muscle in the neck. ${ }^{8}$ Although IMH show an equal sex distribution yet masseteric IMH surely has a male predominance. ${ }^{9}$ Our case was a female patient having an IMH in the masseter muscle making it all the more rare. The common complaint in IMH is usually an asymptomatic slow growing mass with no aesthetic concerns and no skin discoloration as in superficial haemangiomas. ${ }^{10}$ In our case too it was an asymptomatic swelling for the last one year.

Intramuscular sinusoidal hemangioma, first described by Calonje and Fletcher, is characterized by thin walled inter-communicating vascular channels arranged in a sinusoidal pattern. ${ }^{1}$ Masson's lesion is a typical finding in IMH and was found in the HPE in our case too as is seen in Fig. 3. Masson's lesion, an intravascular papillary endothelial hyperplasia is a reactive condition representing an exuberant organization and recanalisation of a thrombus, first described Pierre Masson in 1923. ${ }^{11}$

Intramuscular hemangiomas represent a challenge on diagnosis as they exhibit few signs on clinical examination. Often times the extent of the lesion is not clinically apparent on examination. Definitive preoperative diagnosis has been reported in less than $8 \%$ of cases. ${ }^{12}$ Definitive diagnosis was possible in our case, only after excision and HPE. Preoperative CT scan and FNAC were inconclusive and because of technical constraints sonography couldn't be done.

\section{Conclusion}

Intramuscular Sinusoidal hemangioma through rare in the head and neck region should be kept in the differential diagnosis of any tumour in the head and neck region.

\section{References}

1. Halawar SS, Venugopal R, Varsha BK, Kavya BM. Intramuscular sinusoidal hemangioma with Masson's lesion. J Oral Maxillofac Path. 2013; 17(2):315-7

2. Watson WL, McCarty WD. Surgery Gynecology and Obstetrics 1940; 71:571-88

3. Beham A, Fletcher CD. Intramuscular angioma. A clinicopathological analysis of 74 cases. Histology 1991; 18:53-9

4. Allen PW, Enzinger FM. Hemangioma of skeletal muscle. An analysis of 89 cases. Cancer 1972; 29: 8-22

5. Gamper TJ, Morgan RF. Vascular anomalies: hemangiomaas, Plastic Reconstructive Surgery 2002; 110:572-85

6. Kushraj T, Chatra L, Shenai P, Rao PK, Shetty SR. Cavernous hemangioma of the buccinators muscle-MRI features. Pacific Journal of Medical Sciences 2013; 12:65-70

7. Bucci T, De Glullo F, Insabato L, et al. cavernous hemangioma of the temporalis muscle: a case report and review of literature. Acta Otorhinolarygologica Italica 2008; 28:83-6

8. Clemis JD, Briggs DR, Changus GW. Intramuscular hemangiomas in the head and neck. Canadian Journal of 
Otolaryngology 1975; 4:339-46

9. Hoehn JG, Farrow GM, Devine KD. Invasive hemangiomas of the head and neck. American Journal of Surgery 1970; 120:495-8

10. Wolf GT, Daniel F, Krause CJ, et al. Intramuscular hemangiomas of the head and neck. Laryngoscope 1985; 95:210-3
11. Pablo MD, Salil S, Meena C, et al. Masson's tumour: differential diagnosis of neck lump in children. International Journal of Pediatric Otolaryngology Extra 2006; 1: 196-9

12. Zengin AZ, Celenk P, Sumer AP. Intramuscular hemangioma presenting with multiple phleboliths: A case report. Oral surgery Oral Medicine Oral Pathology Oral radiology 2013; 115:e32-6. 